\title{
Goal-directed therapy: hit early and personalize!
}

\author{
Bernd Saugel $^{1} \cdot$ Frederic Michard $^{2} \cdot$ Thomas W. L. Scheeren $^{3}$
}

Received: 22 June 2017 / Accepted: 22 June 2017 / Published online: 26 June 2017

(C) Springer Science+Business Media B.V. 2017

The serial or continuous assessment of hemodynamic variables_-called hemodynamic monitoring_-allows the evaluation of changes in cardiovascular dynamics and is recommended in patients at risk of hemodynamic instability in perioperative and intensive care medicine [1-3]. Aiming to achieve specific target values of hemodynamic variables is referred to as "goal-directed therapy" (GDT). Whether performing GDT enables patient outcome to be improved is a matter of ongoing scientific evaluation and debate. Therefore, Cronhjort and colleagues should be commended for having performed a systematic review and meta-analysis on the impact of protocolized GDT on mortality in adult critically ill patients [4]. The authors analyzed 13 randomized, clinical, open-label trials including patients treated in the intensive care unit (ICU), emergency department, or "corresponding level of care", only 6 of which were high quality trials with low risk of bias [4]. To be eligible for inclusion in their meta-analysis, studies needed to define its control group as standard of care (including measurement of central venous pressure) without any structured hemodynamic

Bernd Saugel

bernd.saugel@gmx.de

Frederic Michard

frederic.michard@bluewin.ch

Thomas W. L. Scheeren

t.w.l.scheeren@umcg.nl

1 Department of Anesthesiology, Center of Anesthesiology and Intensive Care Medicine, University Medical Center Hamburg-Eppendorf, Martinistrasse 52, 20246 Hamburg, Germany

2 MiCo, Chemin de Chapallaz 4, 1135 Denens, Switzerland

3 Department of Anesthesiology, University Medical Center Groningen, University of Groningen, Hanzeplein 1, 9713 GZ Groningen, The Netherlands intervention [4]. The authors revealed no clinically relevant or statistically significant reduction in all-cause mortality with GDT [4].

Several aspects of the systematic review and meta-analysis warrant further discussion as they allow deriving rules on how to apply GDT in different clinical settings:

1. Hit early This systematic review and meta-analysis investigated a very inhomogeneous group of patients with sepsis, trauma, burn, or undergoing high-risk surgery. Such heterogeneity might limit the usefulness of pooling data [5]. The authors explicitly excluded studies with ICU patients in whom GDT was started "before or in the operating room", i.e., before or during general anesthesia or surgical trauma. Thus, the studies included in this systematic review and meta-analysis have one thing in common: GDT was initiated rather late, i.e. after the "first hit" (surgical trauma or onset of acute illness) had already occurred. However, for GDT to be effective, early timing of the intervention is key. We should consider GDT as a preventive measure to avoid development of organ dysfunction rather than a treatment strategy for established organ failure [6] (Fig. 1). In this respect, there is better evidence for a beneficial impact of GDT on patient outcome in surgical patients than in non-surgical ICU patients [7].

2. Personalize Studies included in the systematic review and meta-analysis were inhomogeneous not only with regard to the patient population and underlying disease leading to ICU treatment but also with regard to the hemodynamic targets used in the study group (cardiac output, stroke volume, stroke volume variation, oxygen delivery, central venous oxygen saturation, or mixed venous oxygen saturation). Comparing studies using such different resuscitation targets in a group of 
Fig. 1 Goal-directed therapy: hit early and personalize. Goal-directed therapy should be initiated early in perioperative and intensive care medicine as a preventive measure to avoid organ failure

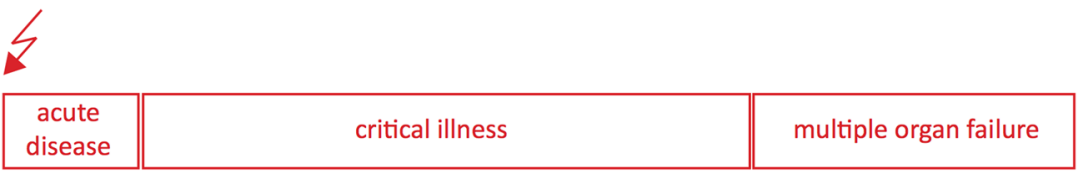

\section{Success of goal-directed therapy}

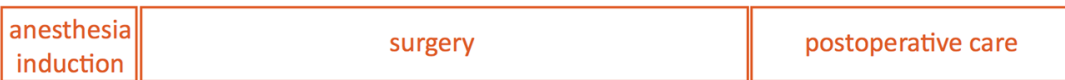

patients suffering from different "ICU syndromes" may be problematic from a physiological point of view. In analogy, in oncology research, one would not try to investigate in a systematic review and meta-analysis whether "chemotherapy" (whatever chemotherapeutic agent used) can cure "cancer" in an inhomogeneous group of patients with leukemia, osteosarkoma, gastrointestinal cancer, lung carcinoma, and glioblastoma. Coming back to GDT, which resuscitation target is the most appropriate depends on the patient population you use it in. For example, in patients with early septic shock and still high central venous oxygen saturation, a resuscitation algorithm with a central venous oxygen saturation target of $70 \%$ can hardly trigger beneficial interventions. To give another example, cardiac output might be better used as a target variable in major abdominal surgery patients than in patients with distributive shock who have (unphysiological) high cardiac output anyway. Therefore, we first need to consider the pathophysiologic alterations causing cardiocirculatory dysfunction in the specific group of patients we intent to treat and then define precisely which hemodynamic variables we want to monitor/ control and what numbers of these variables should be targeted.

A GDT approach can only be as good as the algorithm used to guide administration of fluids and vasoactive agents. This has been discussed many times before $[5,8,9]$ and it is impossible to agree on a "universal treatment algorithm". The challenge is to combine two concepts that seem to be contradictory at first sight: personalized hemodynamic treatment for the individual patient and protocolized care [10]. Although it has been stated a long time ago that using protocols does not ensure excellence, [11] protocolizing hemodynamic care to a certain degree seems to be necessary to rationalize therapy. To help building a symbiosis between "personalization" and "protocolization"-and thus to perform "personalized GDT"-we should use hemodynamic multimodal treatment algorithms [12] and targets adapted to the clinical situation and to the individual patients [10,13] (Fig. 1).

3. Use the right tool The success of GDT therapy also depends on the ability of a hemodynamic monitoring technology to accurately and precisely measure the hemodynamic variable it is supposed to measureor at least track the direction of changes of this variable in response to therapy [14]. This, however, is not a problem of GDT per se but is a technological issue that needs to be addressed in solid validation studies appropriately applying the available statistical methods [15-20]. What is the most appropriate hemodynamic monitoring technique depends on the specific clinical setting it is used in and the hemodynamic variables that are targeted.

4. Choose the right endpoint Last but not least, the effect of any intervention on a certain endpoint measure (e.g. mortality) depends on how frequently this outcome occurs. As ICU mortality has already markedly decreased over time because many aspects of care for critically ill patients have improved, the effect of any single intervention on mortality becomes more and more difficult to prove. In this respect, when mortality rates are low (e.g. after elective surgery), other outcomes such as post-operative complications, hospital length of stay and costs, as well as functional recovery are more appropriate endpoints.

In summary, GDT should be applied as early as possible, at best as a preventive measure to avoid postoperative complications in surgical patients and organ failure in the ICU (Fig. 1). Finally, GDT and its target variables must be tailored to well-defined clinical scenarios and adapted to the individual patient, thus resulting in "personalized GDT". 


\section{Compliance with ethical standards}

Conflict of interest BS collaborates with Pulsion Medical Systems SE (Feldkirchen, Germany) as a member of the medical advisory board and received honoraria for giving lectures and refunds of travel expenses from Pulsion Medical Systems SE. BS received research support from Edwards Lifesciences (Irvince, CA, USA). BS received institutional research grants, unrestricted research grants, and refunds of travel expenses from Tensys Medical Inc. (San Diego, CA, USA). BS received honoraria for giving lectures and refunds of travel expenses from CNSystems Medizintechnik AG (Graz, Austria). FM has been a consultant to Pulsion Medical Systems SE and UP-MED, and an employee of Edwards Lifesciences (VP Global Medical Strategy). He is the founder and managing director of MiCo Sarl, a Swiss consulting firm providing services to medtech companies, digital health startups, and lifescience investors. TWLS received honoraria from Edwards Lifesciences and Masimo Inc. (Irvine, CA, USA) for consulting and for giving lectures. TWLS received honoraria from Pulsion Medical Systems SE for giving lectures. TWLS is associate editor of the Journal of Clinical Monitoring and Computing but had no role in the handling of this manuscript.

Informed consent Not applicable.

Research involving human participants and/or animals Not applicable.

\section{References}

1. Cecconi M, De Backer D, Antonelli M, Beale R, Bakker J, Hofer C, Jaeschke R, Mebazaa A, Pinsky MR, Teboul JL, Vincent JL, Rhodes A. Consensus on circulatory shock and hemodynamic monitoring. Task force of the European Society of Intensive Care Medicine. Intensive Care Med. 2014;40:1795-815. doi:10.1007/ s00134-014-3525-z.

2. Vincent JL, Pelosi P, Pearse R, Payen D, Perel A, Hoeft A, Romagnoli S, Ranieri VM, Ichai C, Forget P, Della Rocca G, Rhodes A. Perioperative cardiovascular monitoring of high-risk patients: a consensus of 12. Crit Care. 2015;19:224. doi:10.1186/ s13054-015-0932-7.

3. Teboul JL, Saugel B, Cecconi M, De Backer D, Hofer CK, Monnet X, Perel A, Pinsky MR, Reuter DA, Rhodes A, Squara P, Vincent JL, Scheeren TW. Less invasive hemodynamic monitoring in critically ill patients. Intensive Care Med. 2016;42:13509. doi:10.1007/s00134-016-4375-7.

4. Cronhjort M, Wall O, Nyberg E, Zeng R, Svensen C, Martensson J, Joelsson-Alm E. Impact of hemodynamic goal-directed resuscitation on mortality in adult critically ill patients: a systematic review and meta-analysis. J Clin Monit Comput. 2017. doi:10.1007/s10877-017-0032-0.

5. Vistisen ST, Keus E, Scheeren TWL. Methodology in systematic reviews of goal-directed therapy: improving but not perfect. Br J Anaesth. 2017. doi:10.1093/bja/aex206.
6. Kern JW, Shoemaker WC. Meta-analysis of hemodynamic optimization in high-risk patients. Crit Care Med. 2002;30:1686-92.

7. Michard F, Giglio MT, Brienza N. Perioperative goal-directed therapy with uncalibrated pulse contour methods: impact on fluid management and postoperative outcome. Br J Anaesth. 2017. doi:10.1093/bja/aex138.

8. Malbrain ML, Reuter DA. Hemodynamic treatment algorithms should follow physiology or they fail to improve outcome. Crit Care Med. 2012;40:2923-4. doi:10.1097/ CCM.0b013e31825f6cd0.

9. Teboul JL, Monnet X, Perel A. Results of questionable management protocols are inherently questionable. Crit Care Med. 2012;40:2536. doi:10.1097/CCM.0b013e3182545759.

10. Saugel B, Vincent JL, Wagner JY. Personalized hemodynamic management. Curr Opin Crit Care. 2017. doi:10.1097/ mcc.0000000000000422.

11. Shoemaker WC. Editorial: protocol medicine. Crit Care Med. 1974;2:279.

12. Molnar Z, Szabo Z, Nemeth M. Multimodal individualized concept of hemodynamic monitoring. Curr Opin Anaesthesiol. 2017;30:171-7. doi:10.1097/ACO.0000000000000440.

13. Saugel B, Huber W, Nierhaus A, Kluge S, Reuter DA, Wagner JY. Advanced Hemodynamic Management in Patients with Septic Shock. BioMed Res Int. 2016;2016:8268569. doi:10.1155/2016/8268569.

14. Clement RP, Vos JJ, Scheeren TWL. Minimally invasive cardiac output technologies in the ICU: putting it all together. Curr Opin Crit Care. 2017. doi:10.1097/mcc.0000000000000417.

15. Squara P, Cecconi M, Rhodes A, Singer M, Chiche JD. Tracking changes in cardiac output: methodological considerations for the validation of monitoring devices. Intensive Care Med. 2009;35:1801-8. doi:10.1007/s00134-009-1570-9.

16. Thiele RH, McMurry TL. Data agnosticism and implications on method comparison studies. Anesth Analg. 2015;121:264-6. doi:10.1213/ane.0000000000000810.

17. Squara P, Imhoff M, Cecconi M. Metrology in medicine: from measurements to decision, with specific reference to anesthesia and intensive care. Anesth Analg. 2015;120:66-75. doi:10.1213/ ane. 0000000000000477 .

18. Montenij LJ, Buhre WF, Jansen JR, Kruitwagen CL, de Waal EE. Methodology of method comparison studies evaluating the validity of cardiac output monitors: a stepwise approach and checklist. Br J Anaesth. 2016;116:750-8. doi:10.1093/bja/aew094.

19. Abu-Arafeh A, Jordan H, Drummond G. Reporting of method comparison studies: a review of advice, an assessment of current practice, and specific suggestions for future reports. Br J Anaesth. 2016;117:569-75. doi:10.1093/bja/aew320.

20. Hapfelmeier A, Cecconi M, Saugel B. Cardiac output method comparison studies: the relation of the precision of agreement and the precision of method. J Clin Monit Comput. 2016;30:149-55. doi:10.1007/s10877-015-9711-x. 